\title{
Catalyst free growth of single-crystalline bismuth nanorods by closed space sublimation technique
}

\author{
WAJEeHa USMAN*, ASghari MAQSOOD \\ Thermal Transport Laboratory, School of Chemical and Materials Engineering, National University of Science and \\ Technology (NUST), Islamabad, Pakistan
}

In the present study, bismuth (Bi) thin films having thickness of $335 \mathrm{~nm}$ have been deposited onto a glass substrate by closed space sublimation (CSS) technique. Besides this, spontaneous growth of Bi nanorods has also been investigated for the first time, without template and catalyst assistance in a substrate temperature range of 380 to $430{ }^{\circ} \mathrm{C}$ using CSS technique. $\mathrm{X}$-ray diffraction (XRD) and scanning electron microscopy (SEM) were employed to investigate microstructure, morphology and roughness of the $\mathrm{Bi}$ nanorods. The diameter and length ranges of $\mathrm{Bi}$ nanorods were 80 to $400 \mathrm{~nm}$ and 3 to $5 \mu \mathrm{m}$, respectively. Moreover, they exhibited a rhombohedral structure with a dominant peak indexed at (012), (104), and (110). The mass percentage of $\mathrm{Bi}$, determined by energy dispersive X-ray (EDX), was $99.93 \%$. The studies of electrical resistivity, Hall coefficient, magnetoresistivity, hole mobility and carrier concentration of Bi thin films were performed at 300 to $350 \mathrm{~K}$ and the electrical properties were found to be a function of temperature. The basic aim was to investigate the spectacular evolution of Bi nanostructures on as-deposited thin films and effects of thickness on their structural, electrical and dielectric properties. Detailed examination of SEM micrographs eliminated all other growth modes except self-catalytic tip growth by Vapor-Solid (VS) growth process which is believed to provide the driving force for spontaneous nanorod growth at high substrate temperature. Deposition of thinner Bi films provided a new possibility for fabrication of Bi nanorods of high quality.

Keywords: crystallite size; bismuth; thin films; nanorods; substrate temperature; template; closed space sublimation

(C) Wroclaw University of Technology.

\section{Introduction}

Spontaneous growth of nanorods (nanowhiskers) on soft metal coatings of indium, cadmium and tin has been studied for a long time [1] but the spectacular evolution of the nanorods has been highly controversial based on the judgment of their proposed growth models. Nanorods having high aspect ratio and one dimensional (1D) material possess widespread applications in electronic transport. In this context, one dimensional (1D) ${ }^{209} \mathrm{Bi}$ isotope is the most intensively studied material due to the ease of understanding quantum confinement, magnetoresistivity and thermoelectric effect [2]. It belongs to a group of $\mathrm{V}$ semimetals with rhombohedral crystal structure [3] and band gap $\left(\mathrm{E}_{\mathrm{g}}\right)$ of $0.038 \mathrm{eV}$ while a slight variation of band gap energy brings

*E-mail: smartbrainz786@gmail.com large changes in electrical properties. Variability from a semimetal to a semiconductor by the size variation makes $\mathrm{Bi}$ nanorods very useful in electronic industry [4]. It also possesses unusual transport properties (large magnetic resistance) due to its highly anisotropic Fermi surface, low carrier density, small carrier effective mass and long carrier mean free path [5].

Contrary to most metals the small effective mass of $-0.001 \mathrm{~m}_{\mathrm{o}}$ and long mean free path of $0.4 \mathrm{~mm}$ at $4 \mathrm{~K}$, makes $\mathrm{Bi}$ nanorods ideal to study quantum confinement effects [6]. Similarly, the elongated Fermi surface leads to Fermi wavelength of $600 \AA$, which is much larger than that of most metals with a few $\AA$, making it ideal for electron transport [7]. A lot of research has been done to fabricate $\mathrm{Bi}$ nanorods (nanowhiskers) using different growth modes like vapor-liquid-solid (VLS) mechanism [8], laser ablation, pulsed laser deposition [9], chemical vapor 
deposition (CVD) [10], sol-gel based growth [11], template-based growth [12], physical vapor deposition (PVD) [13].

The history of Bi nanorods fabrication is dated 20 years ago [14]. The first such structures had a diameter in the range of microns while in the later ones, a diameter of $200 \mathrm{~nm}$ was achieved by high pressure casting. Template assisted method with a porous alumina template has been used to fabricate $\mathrm{Bi}$ nanorods with fine diameters [15], The template-pores were filled with liquid $\mathrm{Bi}$ by high pressure injection technique to get continuous nanorods with small pore size [18]. Thermally induced stress driven mechanism was employed to grow Bi whiskers [19].

Although excellent results can be obtained by MBE (Molecular Beam Epitaxy) and CVD (Chemical Vapor Deposition) methods, they are not cost effective; the requirement of expensive precursors and low growth rate excluded them from our research. CSS method fulfills our major requirements by maintaining low fabrication costs, crystalline morphology control, purity control and large area manufacturing [16]. It provides high crystalline growth rate as the distance between the source and the substrate is minimum, thus high quality crystalline growth is possible with minimum loss of source material [17].

Template and catalyst free RF magnetron sputtering methods were utilized in the past to get $\mathrm{Bi}$ nanorods on Bi thin films at elevated substrate temperature of $200{ }^{\circ} \mathrm{C}$ [8]. Spontaneous growth of $\mathrm{Bi}$ nanostructures by PVD method was studied at high substrate temperature of $110{ }^{\circ} \mathrm{C}$ to $140{ }^{\circ} \mathrm{C}$ [18]. So far, there have been no reports in the literature on the synthesis of Bi nanorods by CSS method.

In this research, we have used CSS method to fabricate Bi thin films and have successfully developed $\mathrm{Bi}$ nanorods of different lengths and diameters at high substrate temperature of $380{ }^{\circ} \mathrm{C}$ to $430{ }^{\circ} \mathrm{C}$ for the first time. Structural characterization and exact growth mechanism of Bi nanorods is also discussed in detail. The growth route of $\mathrm{Bi}$ nanorods by utilizing this simple technique of CSS will definitely open the doors for its application as an efficient sensor.

\section{Experimental}

\subsection{Fabrication of $B i$ thin films}

Thin films were obtained using a closed space sublimation unit (installed at Thin Film Technology (TFT) Research Laboratory COMSATS, Islamabad). Soda lime glass slides having dimensions of $25 \mathrm{~mm} \times 75 \mathrm{~mm}$ were used as substrates. The glass substrates were ultrasonically cleaned by using isopropyl alcohol bath before fabrication of Bi films for $30 \mathrm{~min}$. Bi powder having (99.98\%) purity (supplied by Sigma Aldrich) was uniformly spread in a graphite boat (source) having dimensions of $25 \mathrm{~mm} \times 70 \mathrm{~mm}$. Two halogen lamps of $1000 \mathrm{~W}$ and $500 \mathrm{~W}$ were utilized to heat the source and the substrate, respectively. Two temperature controllers, which were attached to the k-type thermocouples, were used to maintain the source and substrate temperatures at $580{ }^{\circ} \mathrm{C}$ and $450{ }^{\circ} \mathrm{C}$, respectively. In order to transport $\mathrm{Bi}$ vapors from the source to substrate, the source was maintained at a higher temperature than the substrate. Mica sheet was used as a source holder and it also acted as a thermal insulator between the source and substrate. The distance between the source and substrate was optimized at $15 \mathrm{~mm}$, and in order to get uniform film thickness and moderate deposition rates this optimal distance between the source and substrate could be varied easily. Vacuum level in the evaporation chamber of $10^{-3} \mathrm{~Pa}$ was attained with the help of an oil diffusion pump backed up by a rotary-vane pump.

Before starting the source and substrate heating, the film fabrication chamber was evacuated for half an hour. The source with Bi powder was heated at a temperature of $580{ }^{\circ} \mathrm{C}$ and the glass substrates were kept at three different temperatures $380{ }^{\circ} \mathrm{C}, 420{ }^{\circ} \mathrm{C}, 430{ }^{\circ} \mathrm{C}$ during five minute deposition. After the deposition process, Bi films of thicknesses $117.65 \mathrm{~nm}, 226.68 \mathrm{~nm}, 335.20 \mathrm{~nm}$ were obtained, respectively. In order to avoid further deposition, the source heater was turned off while the substrate heater was maintained at the deposition temperature for half an hour. To avoid oxidation of films, the Bi films were kept in vacuum till the source temperature decreased to room 
temperature. The obtained transparent grayishwhite colored thin films were annealed to improve the crystallinity and assure proper diffusion of $\mathrm{Bi}$ atoms at different temperatures of 350, 400 and $450{ }^{\circ} \mathrm{C}$, in the vacuum of $8.5 \times 10^{-3} \mathrm{~Pa}$. The annealed films had good adhesion to the glass substrate, as tested by squash tape test [19]. After annealing, crystallite size was not affected much; more detailed effects were studied through XRD and SEM.

\subsection{Characterization}

Crystalline structure of pure Bi films was examined with PAN analytical spectrometer model (X'pert Pro). X-ray diffraction (XRD) data were collected at an operational voltage of $40 \mathrm{keV}$, using $\mathrm{Cu}-\mathrm{K} \alpha$ line $(\lambda=1.504 \AA$ ) radiation at (SCME), NUST. The surface morphology was examined using a Jeol scanning electron microscopy (SEM) with energy variation (10 keV to $20 \mathrm{keV})$, along with energy dispersive X-ray (EDX) for elemental analysis. The surface roughness of the deposited films was studied by AFM. The atomic force microscopy (AFM) micrographs and thickness calculations were performed by using tipscratch method. Hall effect measurement system (ECOPIA HMS 5000) was utilized to measure the electrical properties such as resistivity, carrier concentration and mobility. For ohmic contacts $\mathrm{InSn}$ was deposited on Bi thin films of various thicknesses, fabricated by CSS method and the measurement was performed at room temperature by using van der Pauw method. LCR impedance analyzer was used to measure dielectric properties that included dielectric constant, dielectric loss and ACconductivity of Bi thin films.

\section{Results and discussion}

\subsection{Structural properties}

The XRD patterns of all the samples are shown in Fig. 1. Bi films deposited on glass substrates at different substrate temperatures of $380{ }^{\circ} \mathrm{C}$, $420{ }^{\circ} \mathrm{C}, 430{ }^{\circ} \mathrm{C}$ exhibit a polycrystalline profile with a dominant peak at (012). The dominant crystallite face is (012) plane, parallel to that of substrate. The diffraction patterns correspond to rhombohedral crystal structure having lattice parameters $(\mathrm{a}=\mathrm{b}=4.55 \AA$ and $\mathrm{c}=11.86 \AA$ and $\alpha=\beta=90^{\circ}$ and $\gamma=120^{\circ}$, which is in agreement with the previously observed results [25]. The (014) and (011) small intensity peaks reveal the preferential crystallite grain growth towards the rhombohedral crystal structure [26].

The crystallite size was determined by Scherrer formula:

$$
D=0.9 \lambda /(\beta \cos \theta)
$$

where $\lambda-\mathrm{X}$ ray wavelength, $\theta-$ Bragg's angle and $\beta$ is the full width at half maximum intensity (FWHM) of the peak which was found to be $30 \mathrm{~nm}$. The average crystallite size dependence on substrate temperature was also studied. The XRD data clearly reveal that when the substrate temperature increases the peak intensity for (012) plane gets more intense. The dominant peak texture can be clearly observed for (012) plane at Ts $=430{ }^{\circ} \mathrm{C}$. It has also been observed that the mean crystallite size increases as the substrate temperature increases due to coalescence of small particles. Similar results have been reported in [20]. The strain and dislocation density decreased causing an improvement in crystallization and decrease in defects as shown in Table 1.

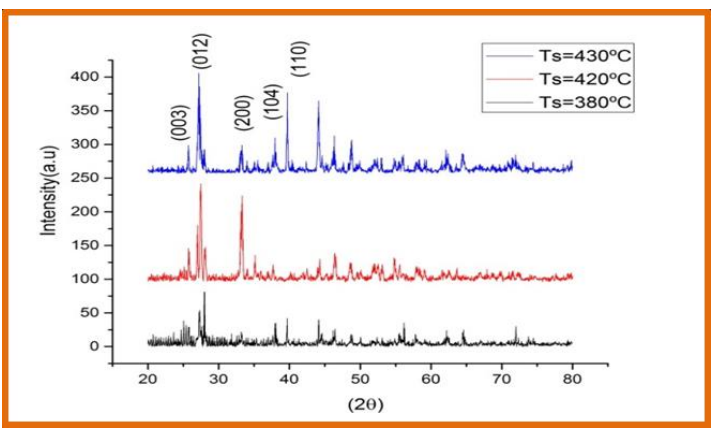

Fig. 1. XRD revealing the effect of substrate temperature on the crystallinity of $\mathrm{Bi}$ thin films.

\subsection{Surface morphology}

The structural characterization of the Bi films by SEM reveals nanorods formation (Fig. 2a, 2b and $2 \mathrm{c}$ ) which has never been reported before at 
Table 1. Structural parameters of pure Bi films deposited at different substrate temperatures.

\begin{tabular}{cccccc}
\hline Samples & $\begin{array}{c}\text { Substrate } \\
\text { temperature }\left({ }^{\circ} \mathrm{C}\right)\end{array}$ & $\begin{array}{c}\text { Lattice } \\
\text { constant }(\AA)\end{array}$ & $\begin{array}{c}\text { Crystallite } \\
\text { size }(\mathrm{nm})\end{array}$ & $\begin{array}{c}\text { Dislocation } \\
\text { density }\left(\times 10^{-4} \mathrm{~nm}^{-2}\right)\end{array}$ & $\begin{array}{c}\text { Strain } \\
\left(\times 10^{-4}\right)\end{array}$ \\
\hline \hline $\mathrm{Bi}-1$ & 380 & 3.2800 & 24.48 & 16.68 & 14.14 \\
$\mathrm{Bi}-2$ & 420 & 3.2720 & 25.70 & 15.14 & 13.99 \\
$\mathrm{Bi}-3$ & 430 & 3.2680 & 32 & 9.765 & 6.193 \\
\hline
\end{tabular}

such high substrate temperature of $380{ }^{\circ} \mathrm{C}$ using CSS method. The average diameter of the nanorods is between 80 to $400 \mathrm{~nm}$ and the length is 3 to $5 \mu \mathrm{m}$. The elemental composition and mass/atomic percentage has been confirmed by EDX analysis. The rhombohedral structure can be observed in the SEM image which shows that during the nanorods formation the rhombohedral symmetry was not disturbed. Bi films were annealed under vacuum at temperatures of 350,400 , and $450{ }^{\circ} \mathrm{C}$ but the nanorods again appeared in SEM images and the thickness of the rods has increased after annealing without disturbance of the structure. Diffused nanorods for bismuth oxide thin films obtained by using thermal deposition had been reported before but no clear morphology was observed [20]. Regarding the growth mechanism there are many theories about spontaneous Bi whiskers formation; (a) the stress driven mechanism [13], (b) screw dislocation model [21], (c) tip grown catalytic VLS/VS growth [22], (d) self catalytic VLS/VS growth mechanism [23], (e) root growth for spontaneous nanorod formation [13].

Considering the possible mechanisms of whiskers growth, the root growth occurs during longer periods of time with residual stress as a driving force. The compressive stress calculated here to be $12 \mathrm{mPa}$ is not sufficient as a possible driving force, in our case. Thus, stress which is believed to be the major cause of nanorods origin is excluded. Similarly, no cracks were observed since the films were of good crystallinity, so in our case intrinsic stress did not play a significant role, contrary to other evaporation methods [14].

SEM micrographs shown in Fig. 3a, 3b clearly reveal the nanorods morphology. After annealing the particle size increases as the thickness of the nanorods increases; the diameter grows up to
$560 \mathrm{~nm}$. The rod morphology can be observed after annealing although their alignment is not uniform and the nanorods are highly disoriented. The crossing over the feature in Fig. 3b clearly reveals that the growth of nanorods did not occur at the same time. Such crossover is important in electronics specially in Josephson junctions [24].

Template and catalyst assisted nanorod growth was quite commonly used, and an early report regarding Bi thin film whiskers obtained using evaporation technique involved deposition of Bi thin film on an as-deposited manganese film which acted as a catalyst. The following post-deposition heating lasted for 3 to $4 \mathrm{~h}$ so the growth of the nanorods was in the presence of Mn layer [25]. In our case we have neither used a template nor a catalyst for nanorod growth. One thing is established that our case is based on non-catalytic approach.

Two famous growth approaches like VaporLiquid-Solid (VLS) and Vapor-Solid (VS) normally involve catalytic growth of semiconductors and elemental nanowires. In VLS, pre-doped gold is assembled to nanodots which catalyze the growth of semiconductor nanowires and float at their ends. Similarly, our Bi nanorods have shown the growth from their own elemental Bi, so a self-catalytic behavior was observed. Likewise, there is a natural tendency in certain materials to grow in the form of prismatic crystals with hexagonal whiskers as reported earlier for zinc oxide $(\mathrm{ZnO})$ [27], so it might be believed that $\mathrm{Bi}$ has such tendency since its hexagonal crystals have a faster growth rate than other crystal systems. SEM results showed hexagonal tips, however, uniaxial symmetry has not been observed in our work. VS growth process, which involves deposition of an element by evaporating it directly, could be considered as the growth process in our case, since the films were 


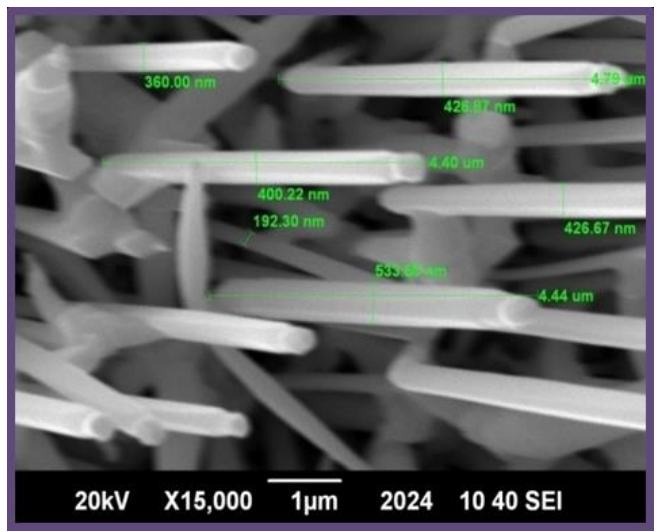

(a)

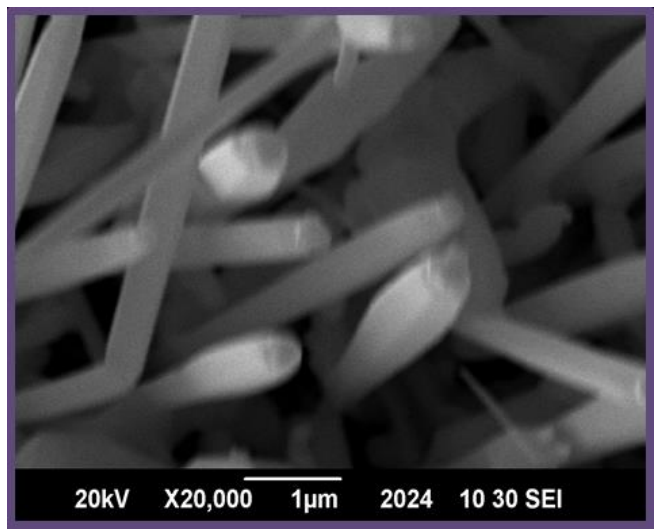

(b)

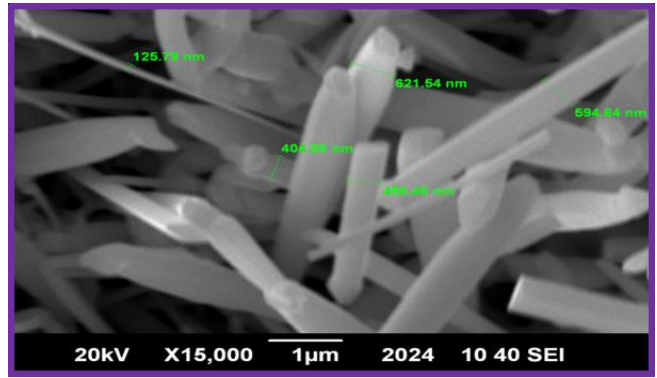

(c)

Fig. 2. SEM micrographs of Bi thin films deposited at different substrate temperatures (a) $430{ }^{\circ} \mathrm{C}$, (b) $420{ }^{\circ} \mathrm{C}$, (c) $380{ }^{\circ} \mathrm{C}$, revealing nanorod morphology of "tip growth process".

deposited by sublimation process. Screw dislocation model, such as in VS or VLS, could not be applied in our case since temperature in thermal evaporation was not as high as in sputtering, secondly, no evidence of screw dislocations was found in SEM, as reported before [18].

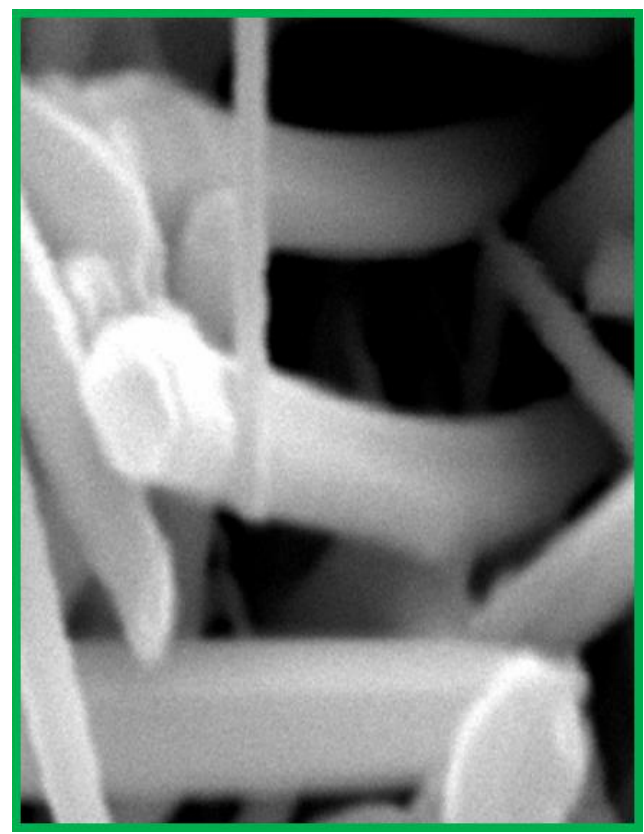

(a)

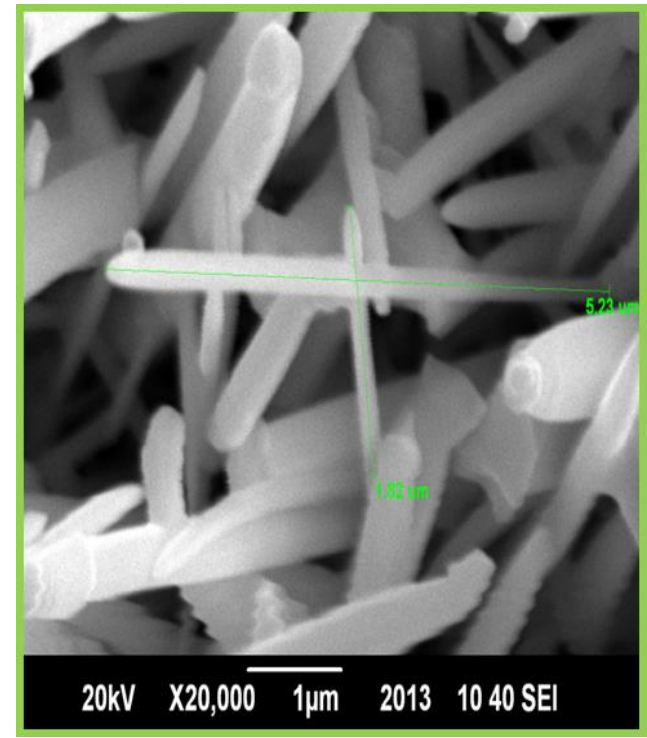

(b)

Fig. 3. SEM micrographs of Bi thin films (a) and (b) revealing tip growth and hexagonal and semi spherical facets with the length exceeding $5 \mu \mathrm{m}$.

After detailed examination of all micrographs and growth conditions it has been established that Bi nanorods are favored due to tip growth by a self-catalytic VS mechanism. It is also evident that Bi seeds are used as catalyst to grow nanowires of semiconductor material as well as catalytic 


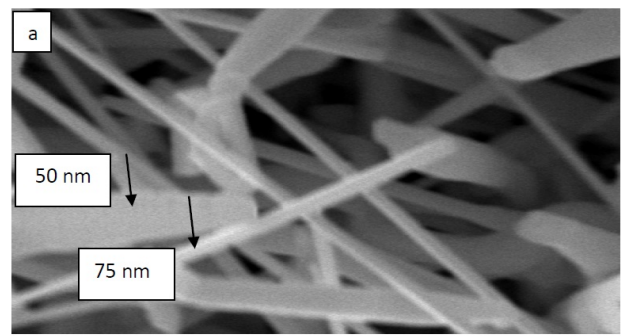

(a)

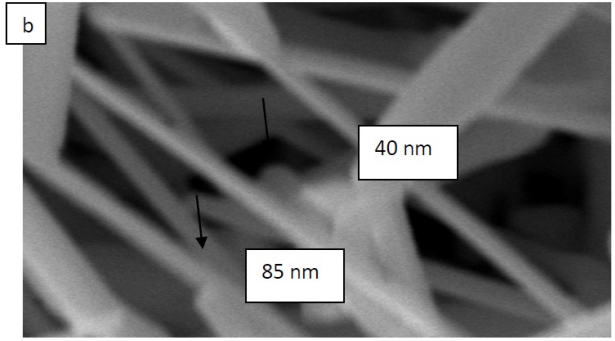

(b)

Fig. 4. (a), (b) SEM micrographs indicating nanowires with thickness of 40, 50, 75, $80 \mathrm{~nm}$.

ability is truly enhanced with $\mathrm{Bi}$. In our case, hexagonal crystal growth rate was faster than in other tip growth modes, which is also confirmed by the presence of a particle or crystal at the tip of our nanorods. A typical shape of these faceted tips is clearly hexagonal. This is supported by the SEM micrographs in Fig. 3a, 3b, 3c as well. Film deposition with decreasing thickness and utilizing a cheap technique of CSS leads to a new route of Bi nanorods fabrication. EDX analysis supports the presence of $\mathrm{Bi}$ as well as the absence of oxides.

During film deposition of thinner films, just below $100 \mathrm{~nm}$, SEM micrographs (Fig. 4) revealed extremely thin nanostructures. Detailed study also showed that the films below $100 \mathrm{~nm}$, deposited by CSS unit, produced films with both whiskers (nanorods) and nanowires. Bi film deposition in changing growth conditions and thicknesses can provide controlled way to grow nanorods and nanowires of high quality. The nanowires appear to be long, straight with crossing over features as shown Fig. 4a. The deposition temperature has also affected the growth of the nanorods but the deposition time played a major role. Thinner films with a clear cross-section were obtained for the films deposited for 15 minutes than for 5 minutes; slow growth of nanostructures enhanced their length in this technique. Thus, by controlling deposition time and thickness, nanowires of high quality can be obtained.

The profilometric analysis carried out by using tip-scratch method revealed the roughness of Bi films. AFM topography of as-deposited Bi thin films did not reveal any porous films and the average height of extended needles was $100 \mathrm{~nm}$. Substrate temperature effect on the roughness of $\mathrm{Bi}$ thin films was also studied; with an increase in substrate temperature the films became more uniform as the roughness of the films decreased [28]. Among the three different substrate temperatures, the films deposited at $430{ }^{\circ} \mathrm{C}$ had the smoothest surface with the rms (root mean square) value of $22.9 \mathrm{~nm}$ as shown in Fig. 5.

\subsection{Elemental composition}

The elemental composition of pure Bi nanorods deposited on glass substrate was determined from EDX measurements with an accelerating voltage of $20 \mathrm{keV}$ and probe current of $1.00 \mathrm{nA}$, in the energy range of 0 to $20 \mathrm{keV}$. The quantitative analysis revealed the presence of dominant amount of $\mathrm{Bi}$ with a mass of $99.93 \%$ and remaining $0.07 \%$ traces of bismuth oxide as shown in Fig. 6. Bi enriched thin films were our basic requirement indicating better adhesion.

\subsection{Electrical properties}

Samples of all Bi thin films with various thicknesses of 110 to $340 \mathrm{~nm}$, prepared in this study, showed positive Hall coefficient and revealed ptype conductivity. Substrate temperature and film thickness affected the electronic properties: a decrease in resistivity with an increase in mobility and carrier concentration was observed in $\mathrm{Bi}$ thin films obtained at higher temperature as shown in Table 2. Electrical resistivity was also found to be a function of film thickness as shown in Fig. 7. When film thickness increased, electrical resistivity decreased due to crystallinity improvement in the thicker films. Compared to RF sputtered $\mathrm{Bi}$ thin films having hole mobility of 


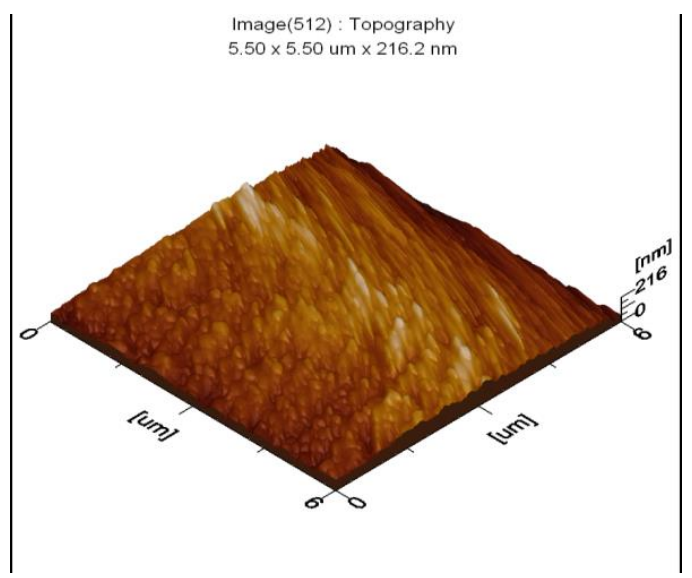

(a)

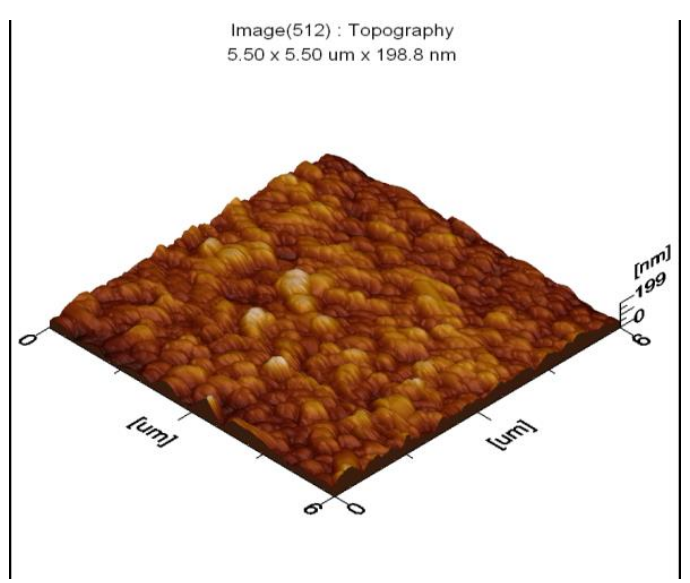

(b)

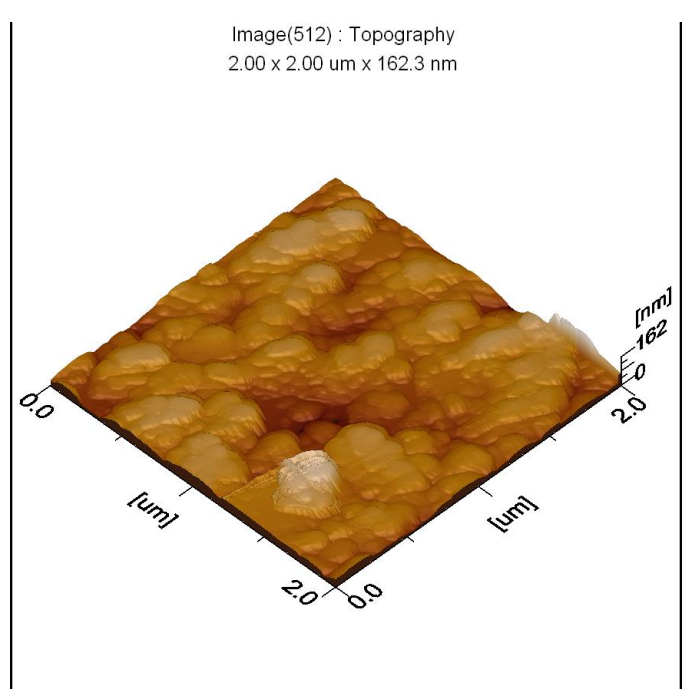

(c)

Fig. 5. AFM top surface morphologies and roughness profiles of Bi films on glass substrate, (a) Bi film at Ts $=380{ }^{\circ} \mathrm{C}$ (b) Bi film at Ts $=420{ }^{\circ} \mathrm{C}$ (c) Bi film at $\mathrm{Ts}=430{ }^{\circ} \mathrm{C}$.

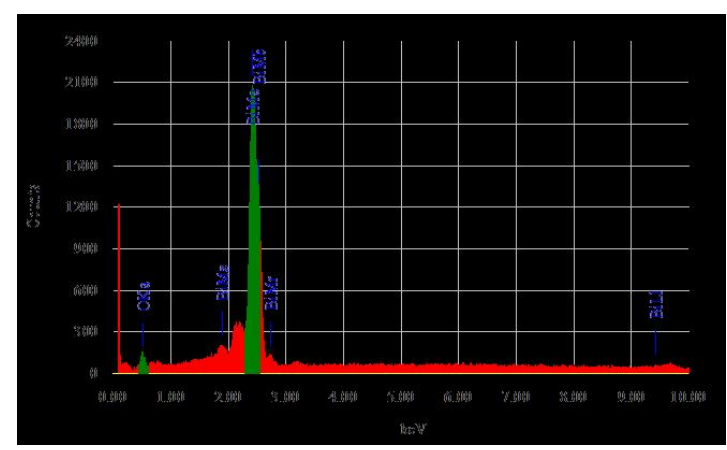

Fig. 6. EDX analysis of Bi nanorod based thin films.

$0.44 \times 10^{2} \mathrm{~cm}^{2} /$ Vs [29], we obtained higher hole mobility which was in a range of $3.664 \times 10^{1}$ to $9.107 \times 10^{2} \mathrm{~cm}^{2} / \mathrm{Vs}$ for Bi thin films deposited by CSS. The electrical resistivity of as-deposited $\mathrm{Bi}$ films was very high, in the range of $2.85 \times 10^{6}$ to $3.89 \times 10^{7} \Omega \cdot \mathrm{cm}$ at a temperature of $300 \mathrm{~K}$, so further research is required to reduce the high resistivity of the films. Compared to the low resistivity of bulk $\mathrm{Bi}$, the thin films have high resistivity which is a direct consequence of finite size effects. The mean free path for electrons is limited by the reduced diameter of the nanowires made on Bi thin films, making them more resistive than bulk bismuth in our case.

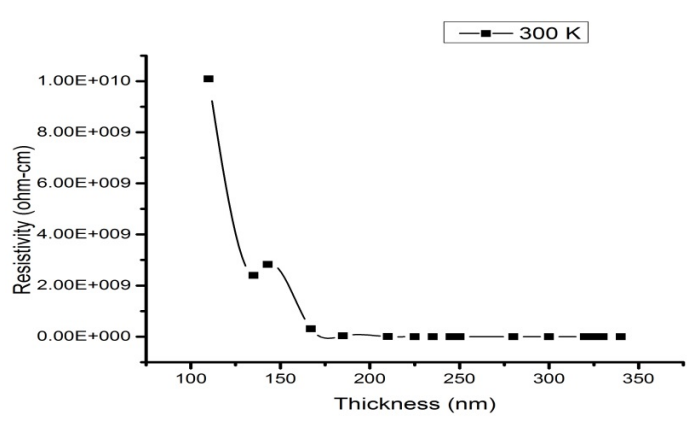

Fig. 7. Resistivity as a function of film thickness.

Hall effect was also studied for thin films of various thickness between 110 to $340 \mathrm{~nm}$ in the temperature range of 300 to $350 \mathrm{~K}$ using the four probe point method with the magnetic field strength of 0.55 T. Magnetoresistance was studied as a function of temperature (Fig. 8) [30] revealing a decreasing trend with an increase in temperature due 
Table 2. Carrier concentration, resistivity and mobility of Bi films deposited at three substrate temperatures (380, 420 and $\left.430{ }^{\circ} \mathrm{C}\right)$.

\begin{tabular}{cccc}
\hline $\begin{array}{c}\text { Substrate } \\
\text { temperature }{ }^{\circ} \mathrm{C}\end{array}$ & $\begin{array}{c}\text { Carrier } \\
\text { concentration }\left(\mathrm{cm}^{-3}\right)\end{array}$ & $\begin{array}{c}\text { Resistivity } \\
(\Omega \cdot \mathrm{cm})\end{array}$ & $\begin{array}{c}\text { Mobility } \\
\left(\mathrm{cm}^{2} / \mathrm{Vs}\right)\end{array}$ \\
\hline \hline 380 & $-5.479 \times 10^{+8}$ & $3.953 \times 10^{+7}$ & $3.664 \times 10^{+1}$ \\
420 & $-1.441 \times 10^{+9}$ & $1.5737 \times 10^{+7}$ & $1.096 \times 10^{+2}$ \\
430 & $-1.688 \times 10^{+9}$ & $2.851 \times 10^{+6}$ & $9.107 \times 10^{+2}$ \\
\hline
\end{tabular}

to an increase in carrier concentration. Hall coefficient decreased as the temperature increased.

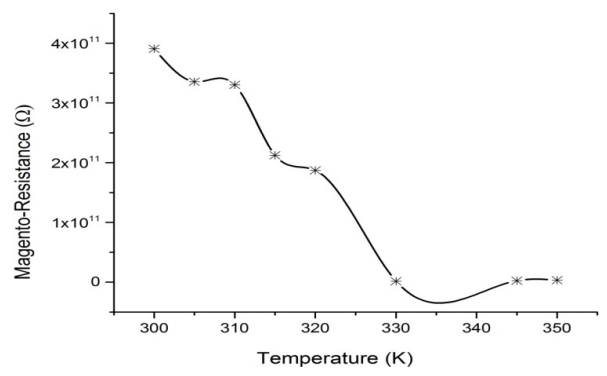

Fig. 8. Magnetoresistance as a function of temperature.

Hall coefficient is an important parameter to explain the type of semiconductor material. Hall coefficient shown in Fig. 9 was calculated using relation 2 and its sign was found to be positive, which showed that holes were the majority charge carriers in the $\mathrm{Bi}$ thin films.

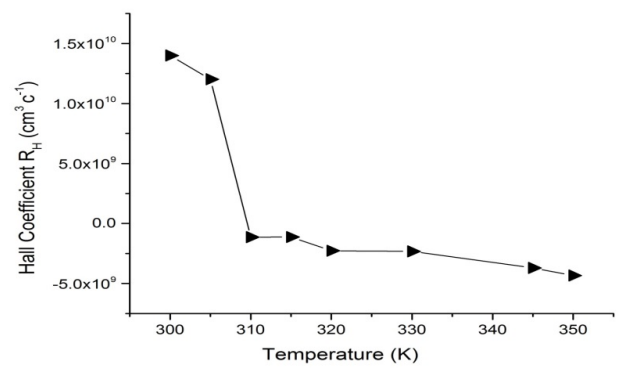

Fig. 9. Hall coefficient as a function of temperature.

For p-type semiconductor, Hall coefficient follows the relation [31]:

$$
R_{H}=\frac{1}{(n e)}
$$

where $\mathrm{n}$ is the carrier concentration and $\mathrm{e}$ is the charge of electron.

Hole mobility values shown in Fig. 10 were calculated from the conductivity measurements from the relation 3 :

$$
\mu=\frac{6}{n e}
$$

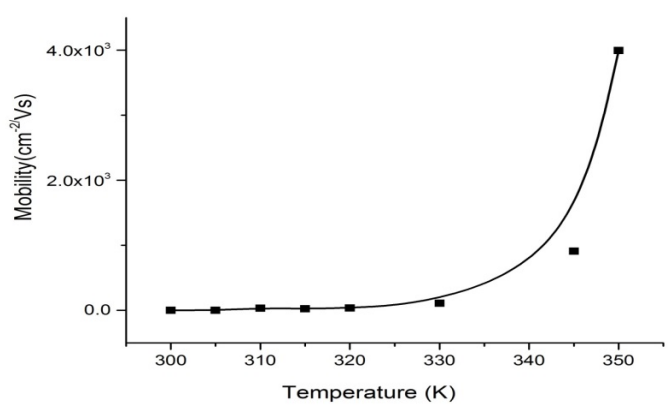

Fig. 10. Hole mobility as a function of temperature.

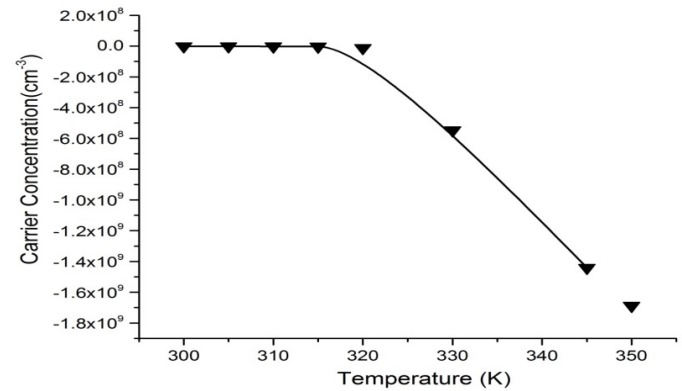

Fig. 11. Carrier concentration as a function of temperature.

It was also found to increase as the temperature increased which is a favorable property for high temperature Hall effect sensors [33]. 
The plot of carrier concentration vs. temperature shown in Fig. 11 shows an increasing trend with temperature.

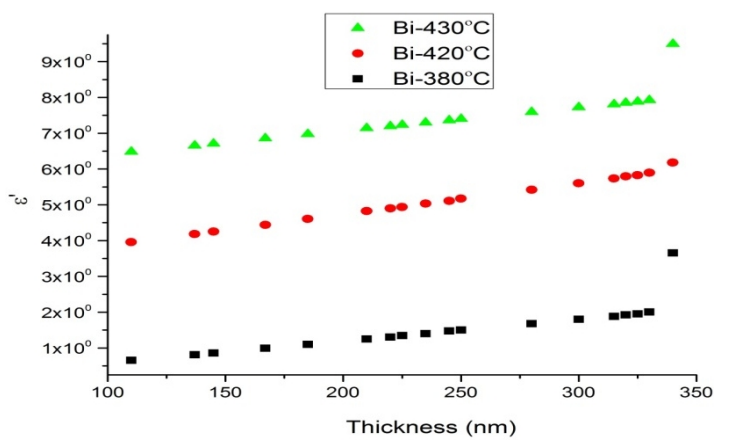

Fig. 12. Dielectric constant as a function of film thickness.

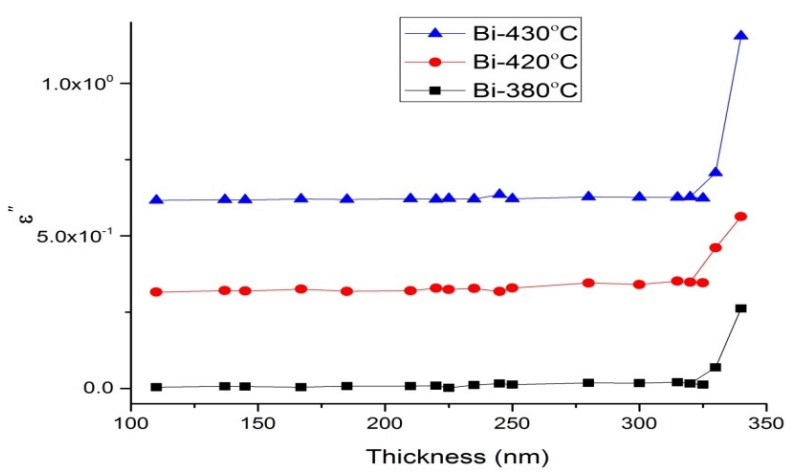

Fig. 13. Dielectric loss as a function of film thickness.

\subsection{Dielectric properties}

LCR impedance analyzer was used to measure the dielectric properties of the Bi thin films at room temperature in the frequency range of $100 \mathrm{~Hz}$ to $5 \mathrm{MHz}$. The dielectric constant $(\varepsilon)$ revealed thickness dependence, showing an increasing trend with the film thickness (Fig. 12). This behavior agrees well with dielectric properties of $\mathrm{Bi}$ oxide thin films [34]. The dielectric loss $\left(\varepsilon^{\text {" }}\right)$ as a function of film thickness was almost constant for all studied films up to the thickness of ca. $325 \mu \mathrm{m}$, then it rose abruptly, as shown in Fig. 13. AC conductivity did not exhibit a linear relationship with film thickness, as shown in Fig. 14, but a gradual rise and fall were observed.

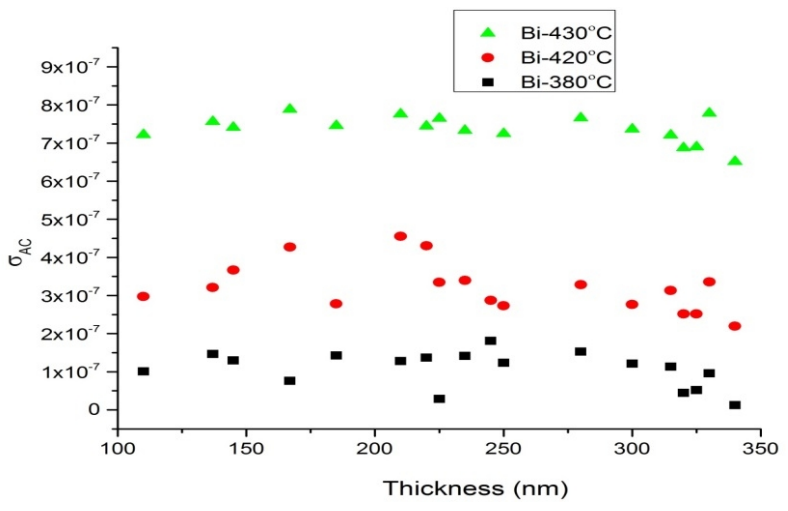

Fig. 14. AC conductivity as a function of film thickness.

\section{Conclusion}

Single crystalline Bi nanorods having diameters of 80 to $400 \mathrm{~nm}$ were fabricated by CSS method. The spectacular evolution of nanorods at high substrate temperature has never been reported before. Structural analysis by XRD confirmed rhombohedral crystal structure and development of nanorods, oriented along (012) direction, whose growth was controlled by the self-catalytic tip growth mechanism due to VS process. The variation in morphology is highly sensitive to substrate temperature as it affects the nucleation and controls the crystallization kinetics. The crystallite size has increased from 24 to $32 \mathrm{~nm}$ with an increase of substrate temperature, producing high quality films, but the real driving force behind spontaneous growth of nanorods was the self-catalytic tip growth mechanism due to VS process. SEM micrographs revealed nanorods and nanowires (heterostructures) as well. An increase in electronic mobility from $3.664 \times 10^{1}$ to $9.107 \times 10^{2} \mathrm{~cm}^{2} / \mathrm{Vs}$ and a decrease in magnetoresistivity was observed with an increase in substrate temperature as well as thickness reduction. In contrast to conventional template, catalyst assisted and thermal evaporation methods, CSS provides a novel and cost effective technique to grow nanorods of high quality which opens the doors for Bi nanorods fabrication and their applications in magnetic, electronic and thermoelectric devices. 


\section{Acknowledgements}

This research was funded by Higher Education Commission (HEC) of Pakistan and all the research work was performed at School of Chemical and Material Engineering (SCME) at National University of Sciences and Technology (NUST) and COMSATS Institute of Information Technology (CIIT), Islamabad, Pakistan.

\section{References}

[1] GALYON G.T., A history of tin whisker theory: 1946 to 2004, in: SMTA International Conference, Chicago, 2004.

[2] Cao Y.Q., Zhu T.J., Zhao X.B., J. Alloy. Compd., 449 (1) (2008), 109.

[3] Duan X., Yang J., Zhu W., Fan X.A., Xiao C., Mater. Lett., 61 (22) (2007), 4341.

[4] Gujar T.P., Shinde V.R., Lokhande C.D., Mane R.S., HAN S.H., Appl. Surf. Sci., 250 (1) (2005), 161.

[5] Dresselhaus M.S., Lin Y.M., Cronin S.B., RaBin O., Black M.R., Dresselhaus G., Koga T., Semicond. Semimet., 71 (2001), 1.

[6] Saini M., Zheng S.H., Wang W., Chien C.L., Morin S., Meet. Abstr., 26 (2011), 1516.

[7] Takayama A., Sato T., Souma S., Oguchi T., TAKAHASHI T., Nano Lett., 12 (4) (2012), 1776.

[8] Cao S., Guo C., Wang Y., Miao J., Zhang Z., LiU Q., Solid State Commun., 149 (1) (2009), 87.

[9] Reppert J., Rao R.,Skove M., He J., Craps M., Tritt T., RaO A.M., Chem. Phys. Lett., 442 (4) (2007), 334.

[10] Kim H.W., LeE J.W., Shim S.H., Sensor. Actuat. BChem., 126 (1) (2007), 306.

[11] Wang Y., XU G., YANG L., Ren Z., Wei X., Weng W., HAN G., Ceram. Int., 35 (4) (2009), 1657.

[12] Huczko A., Appl. Phys. A-Mater., 70 (4) (2000), 365

[13] Stanley S.A., Stuttle C., Caruana A.J., CropPer M.D., Walton A.S.O., J. Phys. D Appl. Phys., 45 (43) (2012), 435304.

[14] Feng L., Mao D., Tang J., Collins R.T., Trefny J.U., J. Electron. Mater., 25 (9) (1996), 1422.

[15] Weber J., Singhal R., Zekri S., Kumar A., Int. Mater. Rev., 53 (4) (2008), 235.

[16] John V., Mahalingam T., Chu J.P., Solid State Electron., 49 (1) (2005), 3.
[17] Ham J., Shim W., OH K.H., Voorhees P.W., LeE W., Appl. Phys. Lett., 98 (4) (2011), 043102.

[18] Ying J.Y., Zhang Z., Zhang L., Dresselhaus M.S., U.S. Patent No. 6,359,288, Washington, DC, 2002.

[19] Yang G., Jung Y., Chun S., Kim D., Kim J., Thin Solid Films, 546 (2013), 375.

[20] Li L., Zhang Y., Li G., Zhang L., Chem. Phys. Lett., 378 (3) (2003), 244.

[21] Mageshwari K., Sathyamoorthy R., Vacuum, 86 (12) (2012), 2029.

[22] Gujar T.P., Shinde V.R., Lokhande C.D., HaN S.H., Mater. Sci. Eng. B-Adv., 133 (1) (2006), 177.

[23] Zhu J., Peng H., Marshall A.F., Barnett D.M., NIX W.D., CUI Y., Nat. Nanotechnol., 3 (8) (2008), 477.

[24] Fanfair D.D., Korgel B.A., Cryst. Growth Des., 5 (5) (2005), 1971.

[25] Zhang Y., He K., Chang C.Z., Song C.L., WAng L.L., Chen X., Xue Q.K., Nat. Phys., 6 (8) (2010), 584.

[26] Hu J.Q., Li Q., Wong N.B., Lee C.S., Lee S.T., Chem. Mater., 14 (3) (2002), 1216.

[27] Mayer L., Rickett R., Stenemann H., J. Appl. Phys., 33 (3) (1962), 982.

[28] Leontie L., Caraman M., Alexe M., Harnagea C., Surf. Sci., 507 (2002), 480.

[29] Kim D.H., LeE S.H., KIM J.K., LeE G.H., Appl. Surf. Sci., 252 (10) (2006), 3525.

[30] Liu K., Chien C.L., Searson P.C., Yu-Zhang K., Appl. Phys. Lett., 73 (10) (1998), 1436.

[31] Fang L., Luo H., Cheng P., Wang Z., Jia Y., Mu G., Wen H.H., Phys. Rev. B, 80 (14) (2009), 140508.

[32] Kaevand T., Kalda J., Lille Ü., Int. J. Renew. Energ. Biofuel., 2013 (2013), 1.

[33] Yang F.Y., LiU K., Hong K., Reich D.H., SEarSON P.C., CHIEN C.L., Science, 284 (5418) (1999), 1335.

[34] Patil S., Puri V.R., Arch. Appl. Sci. Res., 3 (2011), 14.

Received 2014-04-01 Accepted 2015-03-24 